\title{
Dietary Habits and Selenium Intake of Residents in Mountain and Coastal Communities in Japan
}

\author{
Yukiko Mryazaki ${ }^{1,2}$, Hiroshi KoyAma ${ }^{2}$, Yoko SASADA ${ }^{3}$, Hiroshi SATOH ${ }^{4}$, \\ Masami NoJIRI ${ }^{5}$ and Shosuke SuzUKI ${ }^{6}$ \\ ${ }^{1}$ Department of Public Health Nursing, Faculty of Nursing, Jobu University, Gunma 370-1393, Japan \\ ${ }^{2}$ Department of Public Health, Gunma University School of Medicine, Maebashi 371-8511, Japan \\ ${ }^{3}$ Morioka Junior College, Iwate 020-0183, Japan \\ ${ }^{4}$ Department of Public Health and Forensic Medicine, Division of Environmental Health Sciences, \\ Tohoku University School of Medicine, Sendai 980-8575, Japan \\ ${ }^{5}$ Department of Gerontology, Graduate School of International Studies, Obirin University, \\ Machida 194-0294, Japan \\ ${ }^{6}$ Gunma Occupational Health Promotion Center, Maebashi 371-0222, Japan
}

(Received July 14, 2003)

\begin{abstract}
Summary We used a Simple Food Frequency Questionnaire (SFFQ) in combination with other dietary approaches to estimate the selenium intake from different food groups based on the average long-term diet, in two rural communities in Japan, one in a mountain area and the other in a coastal area. The intake frequencies of rice and wheat products were significantly different in the two districts. The intake frequencies of fish, meat, and eggs, which are rich in selenium, were not significantly different. The mean dietary selenium intake, estimated from the SFFQ and the 24-h recall method, was $82.7 \mu \mathrm{g} / \mathrm{d}(n=234)$ (range 19.2$180.1 \mu \mathrm{g} / \mathrm{d}$ ) in the mountain community. The mean dietary selenium intake estimated from the SFFQ and average value of the normal portion size was $118.0 \mu \mathrm{g} / \mathrm{d}(n=123)$ (range $22.6-255.3 \mu \mathrm{g} / \mathrm{d}$ ) in the coastal community. These estimated mean values exceeded the Japanese RDA, although the range of daily selenium intake was large. In the mountain community, fish made the largest contribution to dietary selenium intake ( $48.2 \%$ of daily total), followed by eggs $(24.3 \%)$, and meat $(17.0 \%)$. In the coastal community, fish accounted for $57.7 \%$ of daily total selenium intake, followed by meat (17.5\%), and eggs (16.1\%). In both districts, the total contribution of rice and wheat products was around $10 \%$. It was found that the contribution of fish to dietary selenium intake was high and the contribution of cereals was low among Japanese.
\end{abstract}

Key Words selenium, food frequency questionnaire, dietary habit, nutrition surveys

Selenium is an essential trace element (1), and human selenium deficiency has been reported as the cause of endemic fatal cardiomyopathy in certain low selenium areas of China (2), and as the cause of muscular pain or discomfort in patients on long-term total parenteral nutrition (TPN) (3).

Selenium has many biological functions in humans. One of the most important effects of selenium is its antioxidant effect (4-6), which helps to prevent cardiovascular disease and cancer. Several epidemiological studies have reported that low selenium levels in serum are associated with the risk of vascular disease (7-11), and the risk of coronary heart disease (CHD) in Japanese subjects $(12,13)$. Low selenium intake has also been associated with the risk of cancer (14-18).

Marked selenium deficiency has not been reported in the Japanese general population. The mean daily selenium intake in Japan was estimated at $104.2 \mu \mathrm{g} / \mathrm{d}$ by Suzuki et al. (19), and $154.7 \mu \mathrm{g} / \mathrm{d}$ by Otsuka et al. (20). These studies are not based on the average long-term

E-mail: miyazaki@jobu.ac.jp diet of individuals. Koyama et al. (21) obtained values of the daily selenium intake in a mountain community by three different methods: the value was estimated at $137.3-199.1 \mu \mathrm{g} / \mathrm{d}$ by the $24-\mathrm{h}$ recall method, and $85.8-94.5 \mu \mathrm{g} / \mathrm{d}$ by a food frequency questionnaire, while the measured value was $113.7 \mu \mathrm{g} / \mathrm{d}$ by the duplicated meal method. Little is known about individual selenium intake and regional differences in selenium intake in Japan.

Selenium bioavailability has a high variability, principally due to the different chemical forms that exist in foods (4-6). It is important to know the contributions of various food groups to the overall dietary intake of selenium. The present Japanese recommended dietary allowances (RDA) take selenium bioavailability into consideration (22). However, data on selenium bioavailability to humans is sparse. Therefore, the selenium bioavailability of different food groups in the RDA is tentative.

Food frequency questionnaires (FFQ) are designed to measure average long-term diet (23), which is important for assessing the relationship between nutrition 
and chronic disease. The reproducibility and validity of such questionnaires has been examined in a number of studies (24-27). The simple food frequency questionnaire (SFFQ) was developed to estimate dietary selenium intake for different food groups (21). By using the SFFQ to determine the frequency of consumption in combination with other methods to quantify portion sizes, it is possible to estimate average dietary selenium intake over the long-term.

In the present study, we conducted the SFFQ and some additional nutrition surveys by interview in two rural communities in Japan with different geographies, climates, and industries. One was Ohasama, which is a mountain community, and the other was Nishiizu, which is a coastal community. We estimated selenium intakes by food groups in the two districts with the SFFQ, and assessed the regional characteristics in the food consumption pattern, and dietary selenium intakes.

\section{SUBJECTS AND METHODS}

Survey site

Ohasama is a mountain community, situated in central Iwate Prefecture. It is about $80 \mathrm{~km}$ from the Sanriku-coast (Fig.1), and is situated on the road from the Sanriku-coast to Morioka city, the capital of Iwate prefecture. The area of this district is $246.61 \mathrm{~km}^{2}, 80 \%$ of

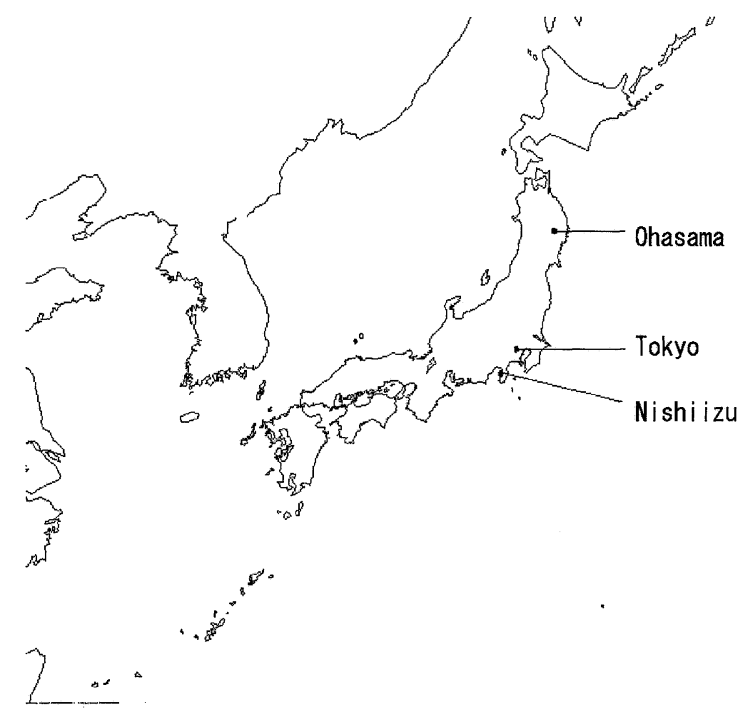

Fig. 1. Map of Japan showing where Ohasama town and the Nishiizu district are located. which is forested mountains. The total population of Ohasama was 6,949 , in 2000. About $28.4 \%$ of the adult population works in agriculture and forestry, $32.9 \%$ works in factories, and $38.7 \%$ works in offices. As of 2000, the major causes of death were cancer, cardiac disease, and cerebrovascular disease (CVD). Mortality due to cancer, cardiac disease, and CVD among the residents was 359.8, 244.6, 187.1 (per 100,000), respectively.

The Nishiizu district includes two rural communities: Nishiizu town $\left(65.88 \mathrm{~km}^{2}\right)$ and Kamo village $\left(39.64 \mathrm{~km}^{2}\right)$. These neighboring communities are situated on the west coast of the Izu Peninsula in Shizuoka prefecture (Fig.1). Most (84\%) of the Nishiizu district is forested. In 2000, the total populations of Nishiizu town and Kamo village were 7,747, and 3,521 respectively. About $9.0 \%$ of the adult population works in agriculture and fishing, $26.1 \%$ works in factories, construction, and the mining industry, and $64.8 \%$ works in service industries and businesses. Previously, the key industry was fishing, but more recently the tourist industry has become the major source of employment in this area. As of 2000, the major causes of death were cancer, cardiac disease, and CVD. Mortality due to cancer, cardiac disease, and CVD among the residents was 337.2, 275.1, 159.7 (per 100,000), respectively.

Subjects

In Ohasama, we interviewed 102 men and 140 women aged 40-69 y. These subjects were selected from participants in a health examination conducted by the government of Ohasama town in 1998, 1999, and 2000. The participants were stratified by sex and age group in each year, and the subjects were randomly selected from each stratum, respectively. The distribution of subjects was 88 (in 1998), 67 (in 1999), 87 (in 2000), and combined data for each year were used. In Nishiizu, we interviewed 55 men aged $44-76$ y and 71 women aged 32-80 y. These subjects participated in a health examination conducted by the government of Nishiizu town and Kamo village in 2000 and volunteered for this study. All subjects gave their signed informed consent.

We excluded eight subjects from analysis for blank on the SFFQ. We also excluded two subjects under the age of 40 and 1 subject of over 80 years. A total of 357 subjects were included in the analysis.

Table 1. Frequency categories and their values converted to daily intake rate.

\begin{tabular}{|c|c|c|c|}
\hline \multicolumn{2}{|c|}{ Major food groups } & \multicolumn{2}{|l|}{ Vegetables } \\
\hline Stated frequency & Value (times/d) & Stated frequency & Value (times/d) \\
\hline Twice or more per day & 2.00 & Two or three times per day & 3.00 \\
\hline Once per day & 1.00 & Twice a day & 2.00 \\
\hline Once every two days & 0.50 & Once a day & 1.00 \\
\hline Once or twice a week & 0.14 & Once every two days & 0.50 \\
\hline Once or twice a month/almost never & 0.04 & Once or twice a week/almost never & 0.14 \\
\hline
\end{tabular}


Table 2. Cereal eating habits and the estimated equivalent daily intake rates.

\begin{tabular}{|c|c|c|c|}
\hline \multicolumn{2}{|c|}{ Cereal eating habits } & \multicolumn{2}{|c|}{ Value (times/day) } \\
\hline Rice & Wheat products & Rice & Wheat products \\
\hline Almost only rice & Less than once or twice a month & 3.0 & 0.0 \\
\hline Almost only rice & Once or twice a week. & 2.7 & 0.3 \\
\hline The rest is rice & Once every two days & 2.5 & 0.5 \\
\hline The rest is rice & Once a day & 2.0 & 1.0 \\
\hline The rest is rice & Twice or more a day & 1.0 & 2.0 \\
\hline
\end{tabular}

\section{Dietary assessment}

Subjects answered the SFFQ by interview. The questionnaire included twelve food group items, and subjects indicated their average frequency of consumption of each item over the past year. The food list consisted of cereals, fish, meat, eggs, soybean products, vegetables, seaweed, milk and dairy products, fruits, wheat products as snacks between meals, cakes and candies, juice and soft drinks with sugar. Questions relating to the intake frequency of major food group items (excluding cereals and vegetables) included five frequency categories from twice or more per day to once or twice a month/almost never (Table 1). The responses were converted into frequencies as daily intake rate (Table 1). For vegetables, the possible responses and frequencies were slightly different (Table 1). For cereals, the frequency of consumption of rice was asked separately from the consumption of wheat products, because the selenium contents in rice and wheat are considerably different. The possible responses and the corresponding frequencies are shown in Table 2.

In Ohasama and Nishiizu, at the same time of the SFFQ, the subjects were shown actual-sized pictures of food items and asked what they ate and the quantity of each food. The same photos were shown in each community. In Ohasama, the subjects were asked to base answers on what they ate the previous day (24-h dietary recall method), while later in Nishiizu, they were asked to base answers on what they usually ate. The averaged consumption of each food item in each community was used as the mean portion size. The daily consumption of each food item was calculated from the mean portion size and daily intake rate.

Daily selenium intake was the sum of the products of the selenium content, the mean portion size, and the daily intake rate of each food (i.e., $\Sigma$ (selenium content $\times$ mean portion size $\times$ daily intake rate)). The food groups of the major dietary selenium sources (21) were used for the estimation. The selenium content of each food group was obtained from the selenium content of foodstuffs measured by Koyama et al. (28). Selenium concentrations in the diet were determined fluorometrically by 2,3-diaminonaphthalene with a spectro-fluorometer (Type 821-FP; Japan Spectroscopic Co., Ltd., Tokyo) after digestion in nitric acid and perchloric acid as described by Watkinson (29). Bovine serum SRM1598 and bovine liver 1577a, produced by NIST (National
Table 3. The selenium content of main food groups.

\begin{tabular}{lc}
\hline \multicolumn{1}{c}{ Food item } & Selenium content $(\mu \mathrm{g} / \mathrm{g})$ \\
\hline Boiled rice & 0.0090 \\
Wheat products & 0.0610 \\
Fish & 0.6287 \\
Meat & 0.6360 \\
Egg & 0.5640 \\
\hline
\end{tabular}

Institute of Standards and Technology, Gaithersburg, $\mathrm{MD})$, was used as a reference material to validate the selenium measurement (28). The selenium contents of main food items are shown in Table 3.

To determine the range of selenium intakes among different individuals, the individual portion size, which was not averaged, was also used for estimation in Nishiizu. For this estimation, the daily intake rate of cereals was modified when subjects took two servings per day. Statistical analysis

The Mann-Whitney U test was used to determine the differences in the frequency of each food item on the SFFQ between the two districts. The significance level was set at $p<0.05$. The analyses were performed using SPSS for Windows (version 11).

\section{RESULTS}

The intake frequencies of rice and wheat products as staple foods in Ohasama and Nishiizu are shown in Table 4. Mean of daily intake rate of rice in Ohasama and Nishiizu was 2.77 and 2.46, respectively. Mean of daily intake rate of wheat products in Ohasama and Nishiizu was 0.24 and 0.54 , respectively. There was a significant difference in the intake frequencies of rice and wheat products between the two districts by the Mann-Whitney U test $(p=0.000)$. Rice was more frequently consumed in Ohasama, and wheat products were more frequently consumed in Nishiizu.

The intake frequencies of other food items are shown in Tables $5(\mathrm{a})-(\mathrm{c})$. When the data for all subjects in a community were combined, the frequencies of consumption of soybean products, vegetables, seaweed, cakes and candies, juice and soft drinks with sugar were significantly greater in Ohasama, and the frequency of consumption of fruits was greater in Nishiizu. The intake frequencies for fish, meat, eggs, milk and dairy 
Table 4. Comparison of food frequency of cereals as staple foods between two districts, Ohasama and Nishiizu (all subjects).

\begin{tabular}{|c|c|c|c|c|c|c|c|c|c|c|}
\hline & \multirow{2}{*}{ District } & \multirow{2}{*}{$n$} & \multicolumn{2}{|c|}{$\begin{array}{l}\text { Mean of daily } \\
\text { intake rate }\end{array}$} & \multicolumn{5}{|c|}{ Frequency categories ${ }^{a}$} & \multirow{2}{*}{$p$ value $^{\mathrm{t}}$} \\
\hline & & & Rice & $\begin{array}{l}\text { Wheat } \\
\text { products }\end{array}$ & 1 & 2 & 3 & 4 & 5 & \\
\hline \multirow[t]{2}{*}{ All subjects } & Ohasama & 234 & 2.77 & 0.24 & $117(50.0 \%)$ & $75(32.1 \%)$ & $21(9.0 \%)$ & $20(8.5 \%)$ & $1(0.4 \%)$ & \multirow[t]{2}{*}{0.000} \\
\hline & Nishiizu & 123 & 2.46 & 0.54 & $35(28.5 \%)$ & $35(28.5 \%)$ & $9(7.3 \%)$ & $36(29.3 \%)$ & $8(6.5 \%)$ & \\
\hline \multirow[t]{2}{*}{ Males } & Ohasama & 98 & 2.81 & 0.19 & $55(56.1 \%)$ & $28(28.6 \%)$ & $9(9.2 \%)$ & $6(6.1 \%)$ & $0(0.0 \%)$ & \multirow[t]{2}{*}{0.002} \\
\hline & Nishiizu & 55 & 2.52 & 0.48 & $20(36.4 \%)$ & $16(29.1 \%)$ & $3(5.5 \%)$ & $12(21.8 \%)$ & $4(7.3 \%)$ & \\
\hline \multirow[t]{2}{*}{ Females } & Ohasama & 136 & 2.73 & 0.27 & $62(45.6 \%)$ & $47(34.6 \%)$ & $12(8.8 \%)$ & $14(10.3 \%)$ & $1(0.7 \%)$ & \multirow[t]{2}{*}{0.000} \\
\hline & Nishiizu & 68 & 2.40 & 0.60 & $15(22.1 \%)$ & $19(27.9 \%)$ & $6(8.8 \%)$ & $24(35.3 \%)$ & $4(5.9 \%)$ & \\
\hline
\end{tabular}

${ }^{a}$ Frequency categories were as follows (cf. Table 2). 1: Almost only rice, and wheat products are less than once or twice a month. 2: Almost rice, and wheat products are once or twice a week. 3: Wheat products are once every two days and the rest is rice. 4: Wheat products are once a day and the rest is rice. 5: Wheat products are twice or more a day and the rest is rice.

${ }^{\mathrm{b}}$ The Mann-Whitney U test was used.

Table 5. Comparison of food frequency between two districts, Ohasama and Nishiizu.

(a) All subjects

\begin{tabular}{|c|c|c|c|c|c|c|c|c|c|}
\hline \multirow{3}{*}{ Food group item } & \multirow{3}{*}{ District } & \multirow{3}{*}{$n$} & \multirow{3}{*}{$\begin{array}{c}\text { Mean of } \\
\text { daily } \\
\text { intake rate }\end{array}$} & \multicolumn{5}{|c|}{ Food frequency categories } & \multirow{3}{*}{$p$ value $^{\mathrm{b}}$} \\
\hline & & & & 1 & 2 & 3 & 4 & 5 & \\
\hline & & & & $\begin{array}{l}\text { Twice or } \\
\text { more per day }\end{array}$ & Once per day & $\begin{array}{l}\text { Once every } \\
\text { two days }\end{array}$ & $\begin{array}{l}\text { Once or } \\
\text { twice a week }\end{array}$ & $\begin{array}{c}\text { Once or twice a } \\
\text { month/almost } \\
\text { never }\end{array}$ & \\
\hline \multirow[t]{2}{*}{ Fish } & Ohasama & 234 & 1.01 & $43(18.4 \%)$ & $123(52.6 \%)$ & $52(22.2 \%)$ & $15(6.4 \%)$ & $1(0.4 \%)$ & \multirow[t]{2}{*}{0.144} \\
\hline & Nishiizu & 123 & 0.97 & $31(25.2 \%)$ & $39(31.7 \%)$ & $31(25.2 \%)$ & $21(17.1 \%)$ & $1(0.8 \%)$ & \\
\hline \multirow[t]{2}{*}{ Meat } & Ohasama & 234 & 0.36 & $1(0.4 \%)$ & $30(12.8 \%)$ & $71(30.3 \%)$ & $106(45.3 \%)$ & $26(11.1 \%)$ & \multirow[t]{2}{*}{0.575} \\
\hline & Nishiizu & 123 & 0.36 & $3(2.4 \%)$ & $11(8.9 \%)$ & $36(29.3 \%)$ & $58(47.2 \%)$ & $15(12.2 \%)$ & \\
\hline \multirow[t]{2}{*}{ Eggs } & Ohasama & 234 & 0.59 & $6(2.6 \%)$ & $75(32.1 \%)$ & $88(37.6 \%)$ & $52(22.2 \%)$ & $13(5.6 \%)$ & \multirow[t]{2}{*}{0.246} \\
\hline & Nishiizu & 123 & 0.56 & $5(4.1 \%)$ & $36(29.3 \%)$ & $34(27.6 \%)$ & $41(33.3 \%)$ & $7(5.7 \%)$ & \\
\hline \multirow[t]{2}{*}{ Soybean products } & Ohasama & 234 & 1.27 & $87(37.2 \%)$ & $105(44.9 \%)$ & $31(13.2 \%)$ & $11(4.7 \%)$ & $0(0 \%)$ & \multirow[t]{2}{*}{0.000} \\
\hline & Nishiizu & 123 & 0.71 & $13(10.6 \%)$ & $38(30.9 \%)$ & $39(31.7 \%)$ & $28(22.8 \%)$ & $5(4.1 \%)$ & \\
\hline \multirow[t]{2}{*}{ Vegetables $^{\mathrm{a}}$} & Ohasama & 234 & 2.58 & $151(64.5 \%)$ & $69(29.5 \%)$ & $11(4.7 \%)$ & $2(0.9 \%)$ & $1(0.4 \%)$ & \multirow{2}{*}{0.001} \\
\hline & Nishiizu & 123 & 2.24 & $62(50.4 \%)$ & $33(26.8 \%)$ & $20(16.3 \%)$ & $7(5.7 \%)$ & $1(0.8 \%)$ & \\
\hline \multirow[t]{2}{*}{ Seaweed } & Ohasama & 234 & 0.70 & $13(5.6 \%)$ & $82(35.0 \%)$ & $98(41.9 \%)$ & $41(17.5 \%)$ & $0(0 \%)$ & \multirow[t]{2}{*}{0.003} \\
\hline & Nishiizu & 123 & 0.58 & $6(4.9 \%)$ & $32(26.0 \%)$ & $44(35.8 \%)$ & $36(29.3 \%)$ & $5(4.1 \%)$ & \\
\hline \multirow[t]{2}{*}{ Milk \& dairy products } & Ohasama & 234 & 0.90 & $42(17.9 \%)$ & $106(45.3 \%)$ & $31(13.2 \%)$ & $38(16.2 \%)$ & $17(7.3 \%)$ & \multirow[t]{2}{*}{0.528} \\
\hline & Nishiizu & 123 & 0.87 & $23(18.7 \%)$ & $51(41.5 \%)$ & $12(9.8 \%)$ & $25(20.3 \%)$ & $12(9.8 \%)$ & \\
\hline \multirow[t]{2}{*}{ Fruits } & Ohasama & 234 & 0.71 & $24(10.3 \%)$ & $81(34.6 \%)$ & $53(22.6 \%)$ & $65(27.8 \%)$ & $11(4.7 \%)$ & \multirow[t]{2}{*}{0.001} \\
\hline & Nishiizu & 123 & 0.89 & $18(14.6 \%)$ & $56(45.5 \%)$ & $29(23.6 \%)$ & $18(14.6 \%)$ & $2(1.6 \%)$ & \\
\hline \multirow{2}{*}{$\begin{array}{l}\text { Wheat products as snacks } \\
\text { between meals }\end{array}$} & Ohasama & 234 & 0.24 & $3(1.3 \%)$ & $26(11.1 \%)$ & $21(9.0 \%)$ & $55(23.5 \%)$ & $129(55.1 \%)$ & \multirow[t]{2}{*}{0.660} \\
\hline & Nishiizu & 123 & 0.22 & $0(0 \%)$ & $12(9.8 \%)$ & $15(12.2 \%)$ & $33(26.8 \%)$ & $63(51.2 \%)$ & \\
\hline \multirow{2}{*}{ Cakes \& candies } & Ohasama & 234 & 0.75 & $34(14.5 \%)$ & $85(36.3 \%)$ & $30(12.8 \%)$ & $42(17.9 \%)$ & $43(18.4 \%)$ & \multirow[t]{2}{*}{0.002} \\
\hline & Nishiizu & 123 & 0.55 & $10(8.1 \%)$ & $35(28.5 \%)$ & $15(12.2 \%)$ & $24(19.5 \%)$ & $39(31.7 \%)$ & \\
\hline \multirow{2}{*}{$\begin{array}{l}\text { Juice \& soft drinks with } \\
\text { sugar }\end{array}$} & Ohasama & 234 & 0.78 & $56(23.9 \%)$ & $52(22.2 \%)$ & $21(9.0 \%)$ & $44(18.8 \%)$ & $61(26.1 \%)$ & \multirow[t]{2}{*}{0.000} \\
\hline & Nishiizu & 123 & 0.52 & $18(14.6 \%)$ & $18(14.6 \%)$ & $10(8.1 \%)$ & $21(17.1 \%)$ & $56(45.5 \%)$ & \\
\hline
\end{tabular}

products, and wheat products as snacks were not significantly different. Among males, significant differences in intake frequency were found for soybean products, vegetables, seaweed, cakes and candies, juice and soft drinks with sugar. These food items were consumed more frequently in Ohasama. Among females, significant differences in intake frequency were found for soybean products, vegetables, fruits, cakes and candies, juice and soft drinks with sugar. These food items, except fruits, were consumed more frequently in Ohasama.

Estimations of daily consumption of the main food items and selenium intakes in Ohasama and in Nishiizu are shown in Tables 6 (a), (b). In Ohasama, the mean daily total selenium intake in males and in females was $96.3 \mu \mathrm{g} / \mathrm{d}$ and $72.9 \mu \mathrm{g} / \mathrm{d}$, respectively. In Nishiizu, the mean daily total selenium intake in males and in females was $125.1 \mu \mathrm{g} / \mathrm{d}$ and $112.3 \mu \mathrm{g} / \mathrm{d}$, respectively. 
(b) Males

\begin{tabular}{|c|c|c|c|c|c|c|c|c|c|}
\hline \multirow{3}{*}{ Food group item } & \multirow{3}{*}{ District } & \multirow{3}{*}{$n$} & \multirow{3}{*}{$\begin{array}{l}\text { Mean of } \\
\text { daily } \\
\text { intake rate }\end{array}$} & \multicolumn{5}{|c|}{ Food frequency categories } & \multirow{3}{*}{$p$ value $^{\mathrm{b}}$} \\
\hline & & & & 1 & 2 & 3 & 4 & 5 & \\
\hline & & & & $\begin{array}{l}\text { Twice or } \\
\text { more per day }\end{array}$ & Once per day & $\begin{array}{l}\text { Once every } \\
\text { two days }\end{array}$ & $\begin{array}{c}\text { Once or } \\
\text { twice a week }\end{array}$ & $\begin{array}{c}\text { Once or twice a } \\
\text { month/almost } \\
\text { never }\end{array}$ & \\
\hline \multirow[t]{2}{*}{ Fish } & Ohasama & 98 & 1.09 & $26(26.5 \%)$ & $45(45.9 \%)$ & $17(17.3 \%)$ & $9(9.2 \%)$ & $1(1.0 \%)$ & \multirow[t]{2}{*}{0.365} \\
\hline & Nishiizu & 55 & 1.01 & $15(27.3 \%)$ & $19(34.5 \%)$ & $11(20.0 \%)$ & $9(16.4 \%)$ & $1(1.8 \%)$ & \\
\hline \multirow[t]{2}{*}{ Meat } & Ohasama & 98 & 0.36 & $1(1.0 \%)$ & $11(11.2 \%)$ & $31(31.6 \%)$ & $48(49.0 \%)$ & $7(7.1 \%)$ & \multirow[t]{2}{*}{0.077} \\
\hline & Nishiizu & 55 & 0.30 & $1(1.8 \%)$ & $4(7.3 \%)$ & $12(21.8 \%)$ & $30(54.5 \%)$ & $8(14.5 \%)$ & \\
\hline \multirow[t]{2}{*}{ Eggs } & Ohasama & 98 & 0.63 & $5(5.1 \%)$ & $32(32.7 \%)$ & $32(32.7 \%)$ & $24(24.5 \%)$ & $5(5.1 \%)$ & \multirow[t]{2}{*}{0.100} \\
\hline & Nishiizu & 55 & 0.52 & $2(3.6 \%)$ & $14(25.5 \%)$ & $15(27.3 \%)$ & $19(34.5 \%)$ & $5(9.1 \%)$ & \\
\hline \multirow[t]{2}{*}{ Soybean products } & Ohasama & 98 & 1.27 & $40(40.8 \%)$ & $35(35.7 \%)$ & $16(16.3 \%)$ & $7(7.1 \%)$ & $0(0 \%)$ & \multirow[t]{2}{*}{0.000} \\
\hline & Nishiizu & 55 & 0.62 & $4(7.3 \%)$ & $16(29.1 \%)$ & $15(27.3 \%)$ & $17(30.9 \%)$ & $3(5.5 \%)$ & \\
\hline \multirow[t]{2}{*}{ Vegetables $^{\mathrm{a}}$} & Ohasama & 98 & 2.55 & $65(66.3 \%)$ & $24(24.5 \%)$ & $6(6.1 \%)$ & $2(2.0 \%)$ & $1(1.0 \%)$ & \multirow[t]{2}{*}{0.009} \\
\hline & Nishiizu & 55 & 2.14 & $27(49.1 \%)$ & $12(21.8 \%)$ & $10(18.2 \%)$ & $5(9.1 \%)$ & $1(1.8 \%)$ & \\
\hline \multirow[t]{2}{*}{ Seaweed } & Ohasama & 98 & 0.74 & $9(9.2 \%)$ & $35(35.7 \%)$ & $34(34.7 \%)$ & $20(20.4 \%)$ & $0(0 \%)$ & \multirow[t]{2}{*}{0.021} \\
\hline & Nishiizu & 55 & 0.56 & $2(3.6 \%)$ & $14(25.5 \%)$ & $21(38.2 \%)$ & $15(27.3 \%)$ & $3(5.5 \%)$ & \\
\hline \multirow[t]{2}{*}{ Milk \& dairy products } & Ohasama & 98 & 0.84 & $19(19.4 \%)$ & $34(34.7 \%)$ & $14(14.3 \%)$ & $19(19.4 \%)$ & $12(12.2 \%)$ & \multirow[t]{2}{*}{0.507} \\
\hline & Nishiizu & 55 & 0.75 & $8(14.5 \%)$ & $21(38.2 \%)$ & $4(7.3 \%)$ & $15(27.3 \%)$ & $7(12.7 \%)$ & \\
\hline \multirow[t]{2}{*}{ Fruits } & Ohasama & 98 & 0.67 & $10(10.2 \%)$ & $33(33.7 \%)$ & $16(16.3 \%)$ & $32(32.7 \%)$ & $7(7.1 \%)$ & \multirow{2}{*}{0.063} \\
\hline & Nishiizu & 55 & 0.79 & $5(9.1 \%)$ & $24(43.6 \%)$ & $16(29.1 \%)$ & $10(18.2 \%)$ & $0(0 \%)$ & \\
\hline \multirow{2}{*}{$\begin{array}{l}\text { Wheat products as snacks } \\
\text { between meals }\end{array}$} & Ohasama & 98 & 0.23 & $0(0 \%)$ & $13(13.3 \%)$ & $8(8.2 \%)$ & $26(26.5 \%)$ & $51(52.0 \%)$ & \multirow[t]{2}{*}{0.706} \\
\hline & Nishiizu & 55 & 0.20 & $0(0 \%)$ & $5(9.1 \%)$ & $6(10.9 \%)$ & $14(25.5 \%)$ & $30(54.5 \%)$ & \\
\hline \multirow[t]{2}{*}{ Cakes \& candies } & Ohasama & 98 & 0.58 & $10(10.2 \%)$ & $24(24.5 \%)$ & $18(18.4 \%)$ & $19(19.4 \%)$ & $27(27.6 \%)$ & \multirow[t]{2}{*}{0.032} \\
\hline & Nishiizu & 55 & 0.42 & $4(7.3 \%)$ & $11(20.0 \%)$ & $3(5.5 \%)$ & $12(21.8 \%)$ & $25(45.5 \%)$ & \\
\hline \multirow{2}{*}{$\begin{array}{l}\text { Juice \& soft drinks } \\
\text { with sugar }\end{array}$} & Ohasama & 98 & 1.01 & $35(35.7 \%)$ & $24(24.5 \%)$ & $5(5.1 \%)$ & $16(16.3 \%)$ & $18(18.4 \%)$ & \multirow[t]{2}{*}{0.010} \\
\hline & Nishiizu & 55 & 0.68 & $13(23.6 \%)$ & $7(12.7 \%)$ & $3(5.5 \%)$ & $14(25.5 \%)$ & $18(32.7 \%)$ & \\
\hline
\end{tabular}

(c) Females

\begin{tabular}{|c|c|c|c|c|c|c|c|c|c|}
\hline \multirow{3}{*}{ Food group item } & \multirow{3}{*}{ District } & \multirow{3}{*}{$n$} & \multirow{3}{*}{$\begin{array}{l}\text { Mean of } \\
\text { daily intake } \\
\text { rate }\end{array}$} & \multicolumn{5}{|c|}{ Food frequency categories } & \multirow{3}{*}{$p$ value $^{\mathrm{b}}$} \\
\hline & & & & 1 & 2 & 3 & 4 & 5 & \\
\hline & & & & $\begin{array}{l}\text { Twice or } \\
\text { more per day }\end{array}$ & Once per day & $\begin{array}{l}\text { Once every } \\
\text { two days }\end{array}$ & $\begin{array}{c}\text { Once or } \\
\text { twice a week }\end{array}$ & $\begin{array}{c}\text { Once or twice a } \\
\text { month/almost } \\
\text { never }\end{array}$ & \\
\hline \multirow[t]{2}{*}{ Fish } & Ohasama & 136 & 0.96 & $17(12.5 \%)$ & $78(57.4 \%)$ & $35(25.7 \%)$ & $6(4.4 \%)$ & $0(0 \%)$ & \multirow[t]{2}{*}{0.196} \\
\hline & Nishiizu & 68 & 0.94 & $16(23.5 \%)$ & $20(29.4 \%)$ & $20(29.4 \%)$ & $12(17.6 \%)$ & $0(0 \%)$ & \\
\hline \multirow[t]{2}{*}{ Meat } & Ohasama & 136 & 0.35 & $0(0 \%)$ & $19(14.0 \%)$ & $40(29.4 \%)$ & $58(42.6 \%)$ & $19(14.0 \%)$ & \multirow[t]{2}{*}{0.468} \\
\hline & Nishiizu & 68 & 0.40 & $2(2.9 \%)$ & $7(10.3 \%)$ & $24(35.3 \%)$ & $28(41.2 \%)$ & $7(10.3 \%)$ & \\
\hline \multirow[t]{2}{*}{ Eggs } & Ohasama & 136 & 0.57 & $1(0.7 \%)$ & $43(31.6 \%)$ & $56(41.2 \%)$ & $28(20.6 \%)$ & $8(5.9 \%)$ & \multirow[t]{2}{*}{0.974} \\
\hline & Nishiizu & 68 & 0.60 & $3(4.4 \%)$ & $22(32.4 \%)$ & $19(27.9 \%)$ & $22(32.4 \%)$ & $2(2.9 \%)$ & \\
\hline \multirow[t]{2}{*}{ Soybean products } & Ohasama & 136 & 1.27 & $47(34.6 \%)$ & $70(51.5 \%)$ & $15(11.0 \%)$ & $4(2.9 \%)$ & $0(0 \%)$ & \multirow[t]{2}{*}{0.000} \\
\hline & Nishiizu & 68 & 0.79 & $9(13.2 \%)$ & $22(32.4 \%)$ & $24(35.3 \%)$ & $11(16.2 \%)$ & $2(2.9 \%)$ & \\
\hline \multirow[t]{2}{*}{ Vegetables $^{\mathrm{a}}$} & Ohasama & 136 & 2.60 & $86(63.2 \%)$ & $45(33.1 \%)$ & $5(3.7 \%)$ & $0(0 \%)$ & $0(0 \%)$ & \multirow[t]{2}{*}{0.027} \\
\hline & Nishiizu & 68 & 2.32 & $35(51.5 \%)$ & $21(30.9 \%)$ & $10(14.7 \%)$ & $2(2.9 \%)$ & $0(0 \%)$ & \\
\hline \multirow[t]{2}{*}{ Seaweed } & Ohasama & 136 & 0.66 & $4(2.9 \%)$ & $47(34.6 \%)$ & $64(47.1 \%)$ & $21(15.4 \%)$ & $0(0 \%)$ & \multirow[t]{2}{*}{0.062} \\
\hline & Nishiizu & 68 & 0.60 & $4(5.9 \%)$ & $18(26.5 \%)$ & $23(33.8 \%)$ & $21(30.9 \%)$ & $2(2.9 \%)$ & \\
\hline \multirow[t]{2}{*}{ Milk \& dairy products } & Ohasama & 136 & 0.95 & $23(16.9 \%)$ & $72(52.9 \%)$ & $17(12.5 \%)$ & $19(14.0 \%)$ & $5(3.7 \%)$ & \multirow[t]{2}{*}{0.937} \\
\hline & Nishiizu & 68 & 0.96 & $15(22.1 \%)$ & $30(44.1 \%)$ & $8(11.8 \%)$ & $10(14.7 \%)$ & $5(7.4 \%)$ & \\
\hline \multirow[t]{2}{*}{ Fruits } & Ohasama & 136 & 0.73 & $14(10.3 \%)$ & $48(35.3 \%)$ & $37(27.2 \%)$ & $33(24.3 \%)$ & $4(2.9 \%)$ & \multirow[t]{2}{*}{0.005} \\
\hline & Nishiizu & 68 & 0.97 & $13(19.1 \%)$ & $32(47.1 \%)$ & $13(19.1 \%)$ & $8(11.8 \%)$ & $2(2.9 \%)$ & \\
\hline \multirow{2}{*}{$\begin{array}{l}\text { Wheat products as snacks } \\
\text { between meals }\end{array}$} & Ohasama & 136 & 0.24 & $3(2.2 \%)$ & $13(9.6 \%)$ & $13(9.6 \%)$ & $29(21.3 \%)$ & $78(57.4 \%)$ & \multirow[t]{2}{*}{0.364} \\
\hline & Nishiizu & 68 & 0.23 & $0(0 \%)$ & $7(10.3 \%)$ & $9(13.2 \%)$ & $19(27.9 \%)$ & $33(48.5 \%)$ & \\
\hline \multirow[t]{2}{*}{ Cakes \& candies } & Ohasama & 136 & 0.87 & $24(17.6 \%)$ & $61(44.9 \%)$ & $12(8.8 \%)$ & $23(16.9 \%)$ & $16(11.8 \%)$ & \multirow[t]{2}{*}{0.013} \\
\hline & Nishiizu & 68 & 0.65 & $6(8.8 \%)$ & $24(35.3 \%)$ & $12(17.6 \%)$ & $12(17.6 \%)$ & $14(20.6 \%)$ & \\
\hline \multirow{2}{*}{$\begin{array}{l}\text { Juice \& soft drinks } \\
\text { with sugar }\end{array}$} & Ohasama & 136 & 0.62 & $21(15.4 \%)$ & $28(20.6 \%)$ & $16(11.8 \%)$ & $28(20.6 \%)$ & $43(31.6 \%)$ & \multirow[t]{2}{*}{0.003} \\
\hline & Nishiizu & 68 & 0.40 & $5(7.4 \%)$ & $11(16.2 \%)$ & $7(10.3 \%)$ & $7(10.3 \%)$ & $38(55.9 \%)$ & \\
\hline
\end{tabular}

${ }^{\text {a }}$ Frequency categories of vegetables are as follows; 1: two or three times per day, 2: twice a day, 3: once a day, 4: once every two days, 5: once or twice a week/almost never (cf. Table1).

$\mathrm{b}$ The Mann-Whitney U test was used. 
Table 6. (a) Estimation of daily consumption of the main food item and selenium intakes in Ohasama.

\begin{tabular}{|c|c|c|c|c|}
\hline Food item & $\begin{array}{l}\text { Mean of daily } \\
\text { intake rate }\end{array}$ & $\begin{array}{l}\text { Mean portion } \\
\quad \text { size }(\mathrm{g})\end{array}$ & $\begin{array}{c}\text { Mean daily } \\
\text { consumption (g) }\end{array}$ & $\begin{array}{l}\text { Mean selenium } \\
\text { intakes }(\mu \mathrm{g})\end{array}$ \\
\hline \multicolumn{5}{|l|}{ Males $(n=98)$} \\
\hline Boiled rice & 2.81 & 260.7 & 731.9 & 6.6 \\
\hline Wheat products as staple foods & 0.19 & 224.0 & 43.2 & 2.6 \\
\hline Wheat products as snacks between meals & 0.23 & 71.9 & 16.6 & 1.0 \\
\hline Fish & 1.09 & 68.8 & 75.0 & 47.2 \\
\hline Meat & 0.36 & 76.5 & 27.7 & 17.6 \\
\hline Eggs $^{b}$ & 0.63 & 60.0 & 37.7 & 21.3 \\
\hline Daily total & & & & 96.3 \\
\hline \multicolumn{5}{|l|}{ Females $(n=136)$} \\
\hline Boiled rice & 2.73 & 172.1 & 470.6 & 4.2 \\
\hline Wheat products as staple foods & 0.27 & 141.7 & 37.6 & 2.3 \\
\hline Wheat products as snacks between meals & 0.24 & 55.4 & 13.3 & 0.8 \\
\hline Fish & 0.96 & 57.6 & 55.2 & 34.7 \\
\hline Meat & 0.35 & 51.9 & 18.3 & 11.6 \\
\hline Eggs $^{b}$ & 0.57 & 60.0 & 34.1 & 19.2 \\
\hline Daily total & & & & 72.9 \\
\hline
\end{tabular}

${ }^{\mathrm{a}}$ Mean portion size was derived from the $24 \mathrm{~h}$ recall method. ${ }^{\mathrm{b}}$ One egg was estimated as $60 \mathrm{~g}$.

(b) Estimation of daily consumption of the main food item and selenium intakes in Nishiizu.

\begin{tabular}{|c|c|c|c|c|}
\hline Food item & $\begin{array}{l}\text { Mean of daily } \\
\text { intake rate }\end{array}$ & $\begin{array}{l}\text { Mean portion } \\
\text { size }(\mathrm{g})\end{array}$ & $\begin{array}{c}\text { Mean daily } \\
\text { consumption }(\mathrm{g})\end{array}$ & $\begin{array}{l}\text { Mean selenium } \\
\text { intakes }(\mu \mathrm{g})\end{array}$ \\
\hline \multicolumn{5}{|l|}{ Males $(n=55)$} \\
\hline Boiled rice & 2.52 & 161.6 & 407.6 & 3.7 \\
\hline Wheat products as staple foods & 0.48 & 190.9 & 91.3 & 5.6 \\
\hline Wheat products as snacks between meals & 0.20 & 114.8 & 23.3 & 1.4 \\
\hline Fish & 1.01 & 122.4 & 124.1 & 78.0 \\
\hline Meat & 0.30 & 98.9 & 29.7 & 18.9 \\
\hline Eggs $^{\mathrm{b}}$ & 0.52 & 60.0 & 30.9 & 17.4 \\
\hline Daily total & & & & 125.1 \\
\hline \multicolumn{5}{|l|}{ Females $(n=68)$} \\
\hline Boiled rice & 2.40 & 112.1 & 269.1 & 2.4 \\
\hline Wheat products as staple foods & 0.60 & 164.4 & 98.4 & 6.0 \\
\hline Wheat products as snacks between meals & 0.23 & 104.2 & 23.7 & 1.4 \\
\hline Fish & 0.94 & 101.9 & 95.4 & 60.0 \\
\hline Meat & 0.40 & 87.4 & 35.0 & 22.2 \\
\hline Eggs $^{\mathrm{b}}$ & 0.60 & 60.0 & 35.9 & 20.2 \\
\hline Daily total & & & & 112.3 \\
\hline
\end{tabular}

${ }^{\mathrm{a}}$ Mean portion size was estimated from the usual average portion size. ${ }^{\mathrm{b}}$ One egg was estimated as $60 \mathrm{~g}$.

Dietary selenium intakes estimated using the SFFQ and the mean portion size in Ohasama are shown in Table 7 (a). When the data for all subjects in a community were combined, the mean daily total selenium intake was $82.7 \mu \mathrm{g} / \mathrm{d}$ (range 19.2-180.1 $\mu \mathrm{g} / \mathrm{d}$ ). The major selenium source was fish, which accounted for $48.2 \%$ of the daily total, followed by eggs $(24.3 \%)$ and meat $(17.0 \%)$. The contributions of rice and wheat products to daily total selenium intake were $6.3 \%$ and $4.0 \%$, respectively.

Dietary selenium intakes estimated using the SFFQ and the mean portion size in Nishiizu is shown in Table 7 (b). When the data for all subjects in a community were combined, the mean daily total selenium intake was $118.0 \mu \mathrm{g} / \mathrm{d}$ (range 22.6-255.3 $\mu \mathrm{g} / \mathrm{d}$ ). The major selenium source was fish, which accounted for $57.7 \%$ of the daily total, followed by meat (17.5\%), eggs (16.1\%). The contributions of rice and wheat products to daily total selenium intake were $2.5 \%$ and $6.1 \%$, respectively.

Dietary selenium intakes estimated using the SFFQ and the individual portion sizes in Nishiizu are shown in Table 7 (c). When the data for all subjects in a community were combined, the mean daily total selenium intake was $123.5 \mu \mathrm{g} / \mathrm{d}$ (range $22.4-422.2 \mu \mathrm{g} / \mathrm{d}$ ). The major selenium source was fish, which accounted for $58.1 \%$ of the daily total, followed by meat (19.1\%), eggs (15.2\%). The contributions of rice and wheat products 
Table 7 .

(a) Dietary selenium intakes estimated using the Simple Food Frequency Questionnaire and mean portion size in Ohasama $(\mu \mathrm{g} / \mathrm{d})$.

\begin{tabular}{|c|c|c|c|c|c|c|}
\hline & $n$ & Mean & SD & Min.-Max. & Median & Percent of daily total \\
\hline All subjects & 234 & & & & & \\
\hline Fish & & 39.9 & 22.5 & $1.7-86.6$ & 36.2 & $48.2 \%$ \\
\hline Eggs & & 20.1 & 14.0 & $1.4-67.7$ & 16.9 & $24.3 \%$ \\
\hline Meat & & 14.1 & 13.5 & $1.3-97.3$ & 6.8 & $17.0 \%$ \\
\hline Rice & & 5.2 & 1.3 & $1.6-7.0$ & 4.6 & $6.3 \%$ \\
\hline Wheat products & & 3.3 & 3.4 & $0.1-20.7$ & 2.7 & $4.0 \%$ \\
\hline Daily total & & 82.7 & 32.9 & $19.2-180.1$ & 79.5 & $100.0 \%$ \\
\hline Males & 98 & & & & & \\
\hline Fish & & 47.2 & 26.7 & $1.7-86.6$ & 43.3 & $49.0 \%$ \\
\hline Eggs & & 21.3 & 16.0 & $1.4-67.7$ & 16.9 & $22.1 \%$ \\
\hline Meat & & 17.6 & 16.3 & $2.0-97.3$ & 6.8 & $18.3 \%$ \\
\hline Rice & & 6.6 & 0.6 & $4.7-7.0$ & 7.0 & $6.9 \%$ \\
\hline Wheat products & & 3.7 & 3.9 & $0.2-15.9$ & 4.3 & $3.8 \%$ \\
\hline Daily total & & 96.3 & 36.2 & $20.0-180.1$ & 95.5 & $100.0 \%$ \\
\hline Females & 136 & & & & & \\
\hline Fish & & 34.7 & 17.1 & $5.1-72.5$ & 36.2 & $47.6 \%$ \\
\hline Eggs & & 19.2 & 12.3 & $1.4-67.7$ & 16.9 & $26.3 \%$ \\
\hline Meat & & 11.6 & 10.4 & $1.3-33.0$ & 4.6 & $15.9 \%$ \\
\hline Rice & & 4.2 & 0.5 & $1.6-4.7$ & 4.2 & $5.8 \%$ \\
\hline Wheat products & & 3.1 & 3.1 & $0.1-20.7$ & 2.7 & $4.3 \%$ \\
\hline Daily total & & 72.9 & 26.3 & $19.2-149.5$ & 65.8 & $100.0 \%$ \\
\hline
\end{tabular}

(b) Dietary selenium intakes estimated using the Simple Food Frequency Questionnaire and mean portion size in Nishiizu $(\mu \mathrm{g} / \mathrm{d})$.

\begin{tabular}{|c|c|c|c|c|c|c|}
\hline & $n$ & Mean & $\mathrm{SD}$ & Min.-Max. & Median & Percent of daily total \\
\hline All subjects & 123 & & & & & \\
\hline Fish & & 68.1 & 48.0 & $3.1-153.9$ & 64.0 & $57.7 \%$ \\
\hline Eggs & & 19.0 & 15.8 & $1.4-67.7$ & 16.9 & $16.1 \%$ \\
\hline Meat & & 20.7 & 22.1 & $2.2-125.9$ & 8.8 & $17.5 \%$ \\
\hline Rice & & 3.0 & 0.9 & $1.0-4.4$ & 2.9 & $2.5 \%$ \\
\hline Wheat products & & 7.2 & 6.2 & $0.3-26.4$ & 5.3 & $6.1 \%$ \\
\hline Daily total & & 118.0 & 57.8 & $22.6-255.3$ & 110.4 & $100.0 \%$ \\
\hline Males & 55 & & & & & \\
\hline Fish & & 78.0 & 52.7 & $3.1-153.9$ & 76.9 & $62.4 \%$ \\
\hline Eggs & & 17.4 & 15.6 & $1.4-67.7$ & 16.9 & $13.9 \%$ \\
\hline Meat & & 18.9 & 22.1 & $2.5-125.9$ & 8.8 & $15.1 \%$ \\
\hline Rice & & 3.7 & 0.8 & $1.5-4.4$ & 3.9 & $2.9 \%$ \\
\hline Wheat products & & 7.0 & 6.7 & $0.3-24.3$ & 4.5 & $5.6 \%$ \\
\hline Daily total & & 125.1 & 60.0 & $29.7-255.3$ & 121.5 & $100.0 \%$ \\
\hline Females & 68 & & & & & \\
\hline Fish & & 60.0 & 42.6 & $9.0-128.1$ & 64.0 & $53.4 \%$ \\
\hline Eggs & & 20.2 & 15.9 & $1.4-67.7$ & 16.9 & $18.0 \%$ \\
\hline Meat & & 22.2 & 22.2 & $2.2-111.2$ & 7.8 & $19.8 \%$ \\
\hline Rice & & 2.4 & 0.5 & $1.0-3.0$ & 2.6 & $2.1 \%$ \\
\hline Wheat products & & 7.4 & 5.8 & $0.3-26.4$ & 6.2 & $6.6 \%$ \\
\hline Daily total & & 112.3 & 55.7 & $22.6-230.4$ & 105.0 & $100.0 \%$ \\
\hline
\end{tabular}

were $2.3 \%$ and $5.2 \%$, respectively.

\section{DISCUSSION}

The mean dietary intake of selenium estimated in this study was similar to the values reported by Otsuka (20), and Koyama (21). It was two or three times higher than the normative requirement (the lower limits of the safe range) estimated by the WHO (4). According to the $\mathrm{WHO}$, the normative requirement is $40 \mu \mathrm{g} / \mathrm{d}$ for males and $30 \mu \mathrm{g} / \mathrm{d}$ for females (aged $18 \mathrm{y}$ and over). Our estimates of dietary intake of selenium also exceeded the Japanese RDA (22), which is 45-55 $\mu \mathrm{g} / \mathrm{d}$ for males and $40-45 \mu \mathrm{g} / \mathrm{d}$ for females (aged $30 \mathrm{y}$ and over). However, the range of daily selenium intakes estimated by the 
(c) Dietary selenium intakes estimated using the Simple Food Frequency Questionnaire and the individual portion size in Nishiizu $(\mu \mathrm{g} / \mathrm{d})$.

\begin{tabular}{|c|c|c|c|c|c|c|}
\hline & $n$ & Mean & $\mathrm{SD}$ & Min.-Max. & Median & Percent of daily total \\
\hline All subjects & 123 & & & & & \\
\hline Fish & & 71.8 & 69.9 & $2.2-330.7$ & 55.0 & $58.1 \%$ \\
\hline Eggs & & 18.8 & 15.8 & $1.4-67.7$ & 16.9 & $15.2 \%$ \\
\hline Meat & & 23.6 & 35.8 & $0.0-232.8$ & 8.1 & $19.1 \%$ \\
\hline Rice & & 2.9 & 1.5 & $0.5-8.1$ & 2.7 & $2.3 \%$ \\
\hline Wheat products & & 6.4 & 6.2 & $0.0-28.1$ & 4.6 & $5.2 \%$ \\
\hline Daily total & & 123.5 & 80.1 & $22.4-422.2$ & 100.0 & $100.0 \%$ \\
\hline Males & 55 & & & & & \\
\hline Fish & & 84.8 & 82.7 & $2.2-330.7$ & 55.0 & $63.4 \%$ \\
\hline Eggs & & 16.9 & 15.5 & $1.4-67.7$ & 16.9 & $12.6 \%$ \\
\hline Meat & & 22.3 & 37.7 & $0.0-232.8$ & 8.1 & $16.7 \%$ \\
\hline Rice & & 3.7 & 1.8 & $0.9-8.1$ & 3.6 & $2.8 \%$ \\
\hline Wheat products & & 6.1 & 6.6 & $0.0-24.9$ & 3.7 & $4.6 \%$ \\
\hline Daily total & & 133.7 & 90.0 & $22.7-337.1$ & 109.1 & $100.0 \%$ \\
\hline Females & 68 & & & & & \\
\hline Fish & & 61.3 & 56.1 & $4.0-264.1$ & 47.2 & $53.2 \%$ \\
\hline Eggs & & 20.2 & 15.9 & $1.4-67.7$ & 16.9 & $17.5 \%$ \\
\hline Meat & & 24.6 & 34.5 & $0.0-190.8$ & 12.0 & $21.4 \%$ \\
\hline Rice & & 2.4 & 0.8 & $0.5-4.1$ & 2.4 & $2.1 \%$ \\
\hline Wheat products & & 6.7 & 5.9 & $0.0-28.1$ & 6.3 & $5.8 \%$ \\
\hline Daily total & & 115.2 & 71.8 & $22.4-422.2$ & 98.2 & $100.0 \%$ \\
\hline
\end{tabular}

SFFQ was large, and selenium intake was low in some subjects.

As shown in Tables 6 (a) and (b), the mean portion size of food items, except eggs, was different between the two districts. It is not clear whether these differences were related to the different method of obtaining portion size or the dietary habit in the two districts. In Ohasama, the 24-h dietary recall method was used to obtain mean portion size. This method can accommodate any level of food description detail, and serves important functions in describing mean values for groups (23). In Nishiizu, subjects were asked the usual portion size. This change in protocol, for portion size information in this study, was made because it was thought to estimate not only portion size as the group mean value, but also individual portion size in Nishiizu. To consider the individual average long-term diet, the usual portion size may be better, because the 24-h dietary recall method depends on the previous day's diet. However, it is possible with both methods to assess group averages.

When individual portion size was used as the amount in Nishiizu, the range of selenium intakes was large. However, the mean of the selenium intakes was not different so much in the use of the mean or individual portion size. This result shows that the food consumption pattern varies among individuals. Selenium intake would probably be low in people who consumed very little fish, meat and eggs. It is not sufficient to estimate the mean value, but it is important to know the dispersion of selenium intakes.

By comparing of the intake frequencies of food items on the SFFQ between Ohasama and Nishiizu, the intake frequencies of fish, meat, and eggs, which are rich in selenium, were not seen to be significantly different. However, the intake frequencies of rice and wheat products were significantly different. The intake frequency of boiled rice was higher in Ohasama than in Nishiizu and the intake frequency of wheat products was lower in Ohasama than in Nishiizu. These differences may be related to regional dietary habits.

The mean selenium intakes were not comparable because of the different methods used to obtain mean portion size, however, the difference in frequency and portion size is reflected in the contribution of rice and wheat products to dietary selenium intake. That is, the contribution of rice to daily selenium intake was greater than the contribution of wheat products to daily selenium intake in Ohasama, and the reverse tendency was observed in Nishiizu.

The mean selenium intake from rice was almost the same in spite of the use of the mean or individual portion size in Nishiizu, and the range of selenium intake from rice was smaller than the range of selenium intake from wheat products. This suggests that rice consumption is less variable than wheat consumption among individual Japanese.

Rice and wheat products made small contributions to dietary selenium intake in both districts, and the values estimated in this study were similar to the values obtained by Otsuka (7.7\%) (20) and Koyama (5-11\%) (21). The proportion of selenium intake that is derived from cereals varies considerably among countries $(4,5$, $30-33$ ), and depends on the content of selenium in the soil, which affects the selenium contents in plants. The mean of selenium content in Japanese rice is $43 \mathrm{ng} / \mathrm{g}$ (range 11-182 ng/g) (34). For comparison, wheat, which is processed into bread and noodles in Japan, and 
which is imported almost entirely from North America, contains $0.30-0.82 \mu \mathrm{g} / \mathrm{g}$ of selenium (35). The low value in rice in Japan is probably due to the soil chemistry.

Even though Ohasama is situated far from the coast, the intake frequency of fish was not significantly different from that at Nishiizu. The contributions of fish to dietary selenium were high in both districts, and were similar to the values reported by Otsuka $(55.5 \%)(20)$ and Koyama (50-60\%) (21).

Levels of selenium in fish are comparatively higher than in other foodstuffs. Though measured values of selenium content in fish demonstrate some range by species, these values do not remarkably differ. Suzuki et al. showed values used for the estimation of selenium intake as $0.875 \mu \mathrm{g} / \mathrm{g}$ (tuna); $0.4 \mu \mathrm{g} / \mathrm{g}$ (flounder); 0.3 $\mu \mathrm{g} / \mathrm{g}$ (sardine); $0.163 \mu \mathrm{g} / \mathrm{g}$ (salmon); $0.3 \mu \mathrm{g} / \mathrm{g}$ (others) (19). The value of selenium content in fish used for the estimation of selenium intake in this study is within the previously reported range, and is nearly the medium value for the selenium content of fish.

Levels of selenium in other foodstuffs shown by Suzuki et al. are as $0.104-0.273 \mu \mathrm{g} / \mathrm{g}$ (meat); $0.447 \mu \mathrm{g} / \mathrm{g}$ (eggs); $0.0148 \mu \mathrm{g} / \mathrm{g}$ (rice); $0.15 \mu \mathrm{g} / \mathrm{g}$ (bread); $0.30 \mu \mathrm{g} /$ $\mathrm{g}$ (noodles) (19). Levels of selenium in meat cereals used in the estimation of selenium intake in this study are slightly lower than these values, eggs and meat are slightly higher. Such variation in the reported values would be due to some reason, for example, the district producing the foodstuffs.

One limitation of this study is that the estimated selenium intake value depends upon the food frequency, portion size, and selenium contents in food groups. It should be carefully considered that the estimated selenium intakes were affected by these values. Further studies, for example, duplicating meal analysis in each district, would be informative for selenium contents and estimation of selenium intakes.

Because fish is a major selenium source in the Japanese diet, the bioavailability of fish selenium is important. Studies have shown marked differences in the ability of selenium from fish to increase selenium status. Some animal studies have shown that the bioavailability of selenium in fish is less than that in wheat (3639). However, selenium from flounder and tuna was found to be more efficient than selenium from other meats at restoring liver glutathione peroxidase activity (40).

Very few selenium bioavailability studies have been conducted with humans. An increased consumption of fish increases plasma selenium concentration, but not the plasma level of glutathione peroxidase or Selenoprotein-P (41). The glutathione peroxidase activities in plasma and erythrocytes were not influenced by shrimp intake (42). In Norwegians, serum and platelet selenium levels were consistently raised by consumption of wheat, but only slightly raised by consumption of fish (43). These findings indicate that the bioavailability of selenium from fish is relatively low in humans. However, selenium from fish intake has been reported to have a marked impact on selenium status in humans (44). The chemical form of selenium in fish is not known, and the reason for the low selenium bioavailability in certain fish is not clear. A possible explanation for low selenium bioavailability in certain fish is that the selenium might combine with other heavy metals, especially mercury, in these fish $(5,45)$.

Wheat selenium is reported to have a high bioavailability, and cereals are an important selenium source in humans $(43,46)$. A greater intake of selenium from cereals may be important for optimal selenium status among the Japanese.

The Japanese RDA assumes that $30 \%$ of dietary selenium is derived from cereals, and that the bioavailability of selenium is $90 \%$ in cereals and $50 \%$ in all other foods, resulting in an overall nutritional availability of dietary selenium of $60 \%$ ( $90 \%$ of $30 \%+50 \%$ of $70 \%$ ) (22). However, because of the low contribution of cereals and the high contribution of fish to selenium intake in the Japanese diet, the nutritional availability of dietary selenium might be lower than the above estimate. Further studies of selenium intake that consider the dietary habits of the Japanese population are needed to estimate the optimal selenium intake.

\section{Acknowledgments}

We thank Ayano Kariya, Nao Kondo, Aiko Morizono, Yuki Kaneko, Naoko Hosokawa, Yuko Miyairi (Gunma University) for their assistance with the dietary survey. We are also grateful to the subjects for their willing cooperation.

\section{REFERENCES}

1) Schwarz K, Foltz CM. 1957. Selenium as an integral part of factor 3 against dietary necrotic liver degeneration. J Am Chem Soc 79: 3292-3293.

2) Yang G, Ge K, Chen J, Chen X. 1988. Selenium-related endemic disease and the daily selenium requirement of humans. Wld Rev Nutr Diet 55: 98-152.

3) Gramm HJ, Kopf A, Brätter P. 1995. The necessity of selenium substitution in total parenteral nutrition and artificial alimentation. J Trace Elements Med Biol 9: 112.

4) WHO. 1996. Selenium. In: Trace Elements in Human Nutrition and Health, p 105-122, WHO, Geneva.

5) Rayman MP. 2000. The importance of selenium to human health. Lancet 356: 233-241.

6) Alarcón M, Martínez MC. 2000. Essentiality of selenium in the human body: relationship with different diseases. Sci Total Environ 249: 347-371.

7) Salonen JT, Alfthan G, Huttunen JK, Pikkarainen J, Puska P. 1982. Association between cardiovascular death and myocardial infarction and serum selenium in a matched-pair longitudinal study. Lancet 2: 175-179.

8) Jackson ML. 1988. Selenium: Geochemical distribution and associations with human heart and cancer death rates and longevity in China and the United States. Biol Trace Elem Res 15: 13-21.

9) Salonen JT, Salonen R, Seppänen K, Kantola M, Parviainen M, Alfthan G, Mäenpää PH, Taskinen E, Rauramaa R. 1988. Relationship of serum selenium and antioxidants to plasma lipoproteins, platelet aggregability 
and prevalent ischaemic heart disease in Eastern Finnish men. Atherosclerosis 70: 155-160.

10) Yeğin A, Yeğin H, Alicigüzel Y, Değer N, Semiz E. 1997. Erythrocyte selenium-glutathione peroxidase activity is lower in patients with coronary atherosclerosis. Jpn Heart J 38: 793-798.

11) Hughes K, Ong C. 1998. Vitamins, selenium, iron, and coronary heart disease risk in Indians, Malays, and Chinese in Singapore. J Epidemiol Community Health 52: 181-185.

12) Deguchi $Y$, Ogata A. 1991. Relationship between serum selenium concentration and atherogenic index in Japanese adults. Tohoku J Exp Med 165: 247-251.

13) Koyama H, Watanabe C, Satoh H, Hosokai H, Tamura S. 1995. Consistent relationship between selenium and apolipoprotein A-II concentrations in the sera of fasting middle-aged male abstainers and regular consumers of alcohol. Biol Trace Elem Res 50: 33-42.

14) Willet WC, Polk BF, Morris JS, Stampfer MJ, Pressel S, Rosner B, Taylor JO, Scheneider K, Hames CG. 1983. Prediagnostic serum selenium and risk of cancer. Lancet 2 (8342): 130-134.

15) Salonen JT, Alfthan G, Huttunen JK, Puska P. 1984. Association between serum selenium and the risk of cancer. Am J Epidemiol 120: 342-349.

16) Clark LC, Combs Jr GF, Turnbull BW, Slate EH, Chalker DK, Chow J, Davis LS, Glover RA, Graham GF, Gross EG, Krongrad A, Lesher Jr JL, Park HK, Sanders Jr BB, Smith CL, Taylor JR. 1996. Effects of selenium supplemention for cancer prevention in patients with carcinoma of the skin. JAMA 276: 1957-1963.

17) Knekt P, Marniemi J, Teppo L, Heliövaara M, Aromaa A. 1998. Is low selenium status a risk factor for lung cancer? Am J Epidemiol 148: 975-982.

18) Yoshizawa K, Willet WC, Morris SJ, Stampfer MJ, Spiegelman D, Rimm EB, Giovanucci E. 1998. Study of prediagnostic selenium level in toenails and the risk of advanced prostate cancer. J Natl Cancer Inst 90: 12191224.

19) Suzuki T, Imai H, Kobayashi K, Hongo T, Kashiwazaki H, Ohtsuka R, Suzuki H, Ishida H. 1988. Dietary intake of selenium in Japanese-An estimation by analyzed and reported values in foodstuffs and cooked dishes. J Jpn Soc Nutr Food Sci 41: 91-102 (in Japanese).

20) Otsuka Y, Isomoto S, Noda H. 2000. Dietary intake of trace elements in the general population, estimated from a regional nutritional survey, and comparison with recommended dietary allowances and tolerable upper intake levels. Jpn J Public Health 47: 809-819 (in Japanese).

21) Koyama H, Kasanuma $Y$, Nakatsuka $H$, Watanabe $C$, Satoh H, Watanabe T. 1996. Measurment of selenium intake in duplicate diets and attempt to develop a short food-frequency questionnaire for assessing dietary selenium intake by food groups. Jpn J Hyg 51: 397 (in Japanese).

22) Ministry of Health and Welfare. 1999. Selemiun. In: The 6th Japanese recommended dietary allowances, $p$ 160-163, Daiichi Shuppan, Tokyo (in Japanese).

23) Willett WC. 1998. Food-frequency methods. In: Nutritional Epidemiology 2nd Edition, p 74-94, Oxford University Press, New York.

24) Willett WC, Sampson L, Stampfer MJ, Rosner B, Bain C, Witschi J, Hennekens CH, Speizer FE. 1985. Reproducibility and validity of a semiquantitative food frequency questionnaire. Am J Epidemiol 122: 51-65.

25) Rimm EB, Giovannucci EL, Stampfer MJ, Colditz GA, Litin LB, Willett WC. 1992. Reproducibility and validity of an expanded self-administered semiquantitative food frequency questionnaire among male health professionals. Am J Epidemiol 135: 1114-1126.

26) Männistö S, Virtanen M, Mikkonen T, Pietinen P. 1996. Reproducibility and validity of a food frequency questionnaire in a case-control study on breast cancer. J Clin Epidemiol 49: 401-409.

27) Hu FB, Rimm E, Smith-Warner SA, Feskanich D, Stampfer MJ, Ascherio A, Sampson L, Willett WC. 1999. Reproducibility and validity of dietary patterns assessed with a food-frequency questionnaire. Am J Clin Nutr 69: 243-249.

28) Miyazaki Y, Koyama H, Nojiri M, Suzuki S. 2002. Relationship of dietary intake of fish and non-fish selenium to serum lipids in Japanese rural coastal community. $J$ Trace Elem Med Biol 16: 83-90.

29) Watkinson JH. 1966. Fluorometric determination of selenium in biological material with 2,3-diaminonaphthalene. Anal Chem 38: 92-97.

30) Klapec T, Mandić ML, Grgić J, Primorac Lj, Ikić M. Lovrić T, Grgić Z, Herceg Z. 1998. Daily dietary intake of selenium in eastern Croatia. Sci Total Environ 217: 127136.

31) Kadrabová J, Mad'acić A, Ginter E. 1998. Determination of the daily selenium intake in Slovakia. Biol Trace Elem Res 61: 277-286.

32) Backović D, Marinković J, Jorga J, Pavlica M, Maksimović Z, Nikolić M. 1999. Environmental factors, health-related habits, and serum selenium levels in cancer patients and healthy controls. Biol Trace Elem Res 67: 55-62.

33) Duffield AJ, Thomson CD. 1999. A comparison of methods of assessment of dietary selenium intakes in Otago, New Zealand. Br J Nutr 82: 131-138.

34) Yoshida M, Yasumoto K. 1987. Selenium contents of rice grown at various sites in Japan. J Food Compos Anal 1: $71-75$.

35) Yoshida M, Yasumoto Y. 1988. Selenium content in wheat and soybean products consumed by Japanese. $J$ Jpn Soc Nutr Food Sci 41: 320-323 (in Japanese).

36) Cantor AH, Scott ML, Noguchi T. 1975. Biological availability of selenium in feedstuffs and selenium compounds for prevention of exudative diathesis in chicks. J Nutr 105: 96-105.

37) Douglass JS, Morris VC, Soares JH Jr, Levander OA. 1981. Nutrition availability to rats of selenium in tuna, beef kidney, and wheat. J Nutr 111: 2180-2187.

38) Alexander AR, Whanger PD, Miller LT. 1983. Bioavailability to rats of selenium in various tuna and wheat products. J Nutr 113: 196-204.

39) Yoshida M, Iwami K, Yasumoto K. 1984. Determination of nutritional efficiency of selelnium contained in processed skipjack meat by comparison with selenite. J Nutr Sci Vitaminol 30: 395-400.

40) Wen HY, Davis RL, Shi B, Chen J-J, Chen L, Boylan M, Spallhols JE. 1997. Bioavailability of selenium from veal, chicken, beef, pork, lamb, flounder, tuna, selenomethionine, and sodium selenite assessed in seleniumdeficient rats. Biol Trace Elem Res 58: 43-53.

41) Huang W, Åkesson B, Svensson BG, Schütz A, Burk RF, Skerfving S. 1995. Selenoprotein P and glutathione peroxidase (EC 1.11.1.9) in plasma as indices of selenium 
status in relation to the intake of fish. Br J Nutr 73: 455-461.

42) Bügel SH, Sandström B, Larsen EH. 2001. Absorption and retention of selenium from shrimps in man. J Trace Elements Med Biol 14: 198-204.

43) Meltzer HM, Bibow K, Paulsen IT, Mundal HH, Norheim G, Holm H. 1993. Different bioavailability in humans of wheat and fish selenium as measured by blood platelet response to increased dietary Se. Biol Trace Element Res 36: 229-241.

44) Hagmar L, Persson-Moschos M, Åkesson B, Schütz A.
1998. Plasma levels of selenium, selenoprotein $\mathrm{P}$ and glutathione peroxidase and their correlations to fish intake and serum levels of thyrotropin and thyroid hormones: A study on Latvian fish consumers. Eur J Clin Nutr 52: 796-800

45) Mutanen M. 1986. Bioavailability of selenium. Ann Clin Res 18: 48-54.

46) Djujić IS, Stankov ON, Milovac M, Janković V, Djermanović V. 2000. Bioavailability and possible benefits of wheat intake naturally enriched with selenium and its products. Biol Trace Element Res 77: 273-285. 